\title{
Recent Results on B Spectroscopy at the Tevatron
}

\author{
Martin Heck \\ for the CDF and D0 Collaborations \\ KIT - Institut für Experimentelle Kernphysik \\ Wolfgang-Gaede-Str. 1, 76131 Karlsruhe - Germany
}

\begin{abstract}
The Tevatron collider at Fermilab provides a rich environment for $B$ spectroscopy. Recently the first direct observation of the $B_{c}$ meson has reduced its mass uncertainty by two orders of magnitude. A search for $\eta_{b}$ mesons provides the best limit on its production in $p \bar{p}$ colliders. In studies on orbitally excited $B_{d}$ mesons for the first time the narrow states could be separated from each other. The orbitally excited $B_{s}$ mesons have been observed for the first time. With the charged $\Sigma_{b}^{(*)}$ a second $B$ baryon could be established beside the $\Lambda_{b}$.
\end{abstract}

\section{Introduction}

The Tevatron is a $p \bar{p}$ collider with an energy of $\sqrt{s}=1.96 \mathrm{TeV}$, capable of producing all $B$ hadron species with a cross section for $b \bar{b}$ production of about $50 \mu \mathrm{b}$. For each experiment, CDF and D0, an integrated luminosity of more than $2.5 \mathrm{fb}^{-1}$ has been delivered, increasing faster than ever before with most parts of the detector working around $80 \%$ of the time. Nevertheless studies are complicated due to a 1000 times higher inelastic cross section, a high number of fragmentation tracks and mainly low transversal momentum for the $b$ quark particles.

These disadvantages force the Tevatron experiments to use triggers, saving only events with special structures.

In each experiment a trigger based on two tracks identified as muons and consistent with the $J / \psi$ mass for decays of $B \rightarrow J / \psi X$ is used and, specific to CDF, one based on two tracks forming a secondary vertex, using the relative longevity of $\mathrm{B}$ hadrons.

The studied particles can probe effective theories in a wide kinematical range, such as HQET, NRQCD, lattice calculations and others.

These proceedings first cover the ground states of $B_{c}$ and $\eta_{b}$, then the orbitally excited states of $B_{d}$ and $B_{s}$.

As baryons the $\Sigma_{b}^{+}$and its isospin partner $\Sigma_{b}^{-}$are covered as well as their spin excited states.

\section{First direct observation of $B_{c}$}

Some years ago the Tevatron experiments reported the first observation of the $B_{c}$ in the semileptonic decay to $J / \psi \mu / e X[2]$. In the semileptonic decay channel it is taken advantage of the low number of leptons in an hadronic environment, but no precise mass measurement can be performed due to at least one missing neutrino.

A recent CDF study [3] in the exclusive decay channel $B_{c} \rightarrow J / \psi \pi^{+}$finds a significant signal, shown in Fig. 1. The mass of this $B_{c}$ signal is measured to be $6276.5 \pm 4.0$ (stat) \pm 2.7 (syst) $\mathrm{MeV} / \mathrm{c}^{2}$. The former measurement in the semileptonic channels an uncertainty of $400 \mathrm{MeV} / \mathrm{c}^{2}$, which means the new measurement is two orders of magnitude better than the old one. With the new measurement, experimental uncertainties are much smaller than theoretical uncertainties. 


\section{$3 \quad$ Search for $\eta_{b}$}

A CDF study [4] on the last undiscovered SM ground state meson, the $\eta_{b}$, is done in the decay channel $\eta_{b} \rightarrow J / \psi J / \psi$. Despite forbidden in leading order NRQCD it can be possible to see a signal in this channel, as the decay $\eta_{c} \rightarrow \Phi \Phi$, which is also forbidden, is seen with a branching ratio of about $1 \%$. Scaling this by $\left\{\frac{m_{c}}{m_{b}}\right\}^{4}$ to account for the difference between $b$ and $c$ quark, one gets a prediction for the yield of $\eta_{b}$ in a specific kinematical range. With cuts applied to enrich the signal and for a well understanding of the efficiency, 0.2 to 20 events are expected at CDF. The experimental result

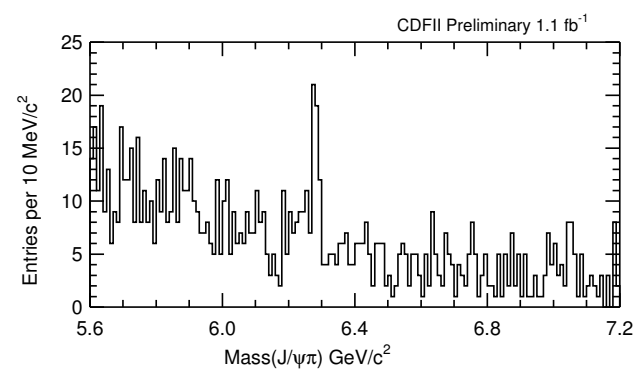

Figure 1: Mass Spectrum of $J / \psi \pi$ with $B_{c}$ signal at about $6.3 \mathrm{GeV} / \mathrm{c}^{2}$. for the $J / \psi J / \psi$ mass spectrum is shown in Fig. 2. From this and the assumption, that the $\eta_{b}$ is about $20 \mathrm{MeV} / \mathrm{c}^{2}$ broad, which is a little less than the $\eta_{c}$, one derives a $95 \%$ confidence limit on the expected number of $\eta_{b}$ for each $\eta_{b}$ mass as shown in Fig. 2, too. The obtained limit is with 7.18 events for an $\eta_{b}$ mass of $9.32 \mathrm{GeV} / \mathrm{c}^{2}$ already inside the predicted range. With other measurements it can be translated to:

$\sigma\left(p \bar{p} \rightarrow \eta_{b} X ;|\eta|<0.6 ; \mathrm{p}_{T}>3 \mathrm{GeV} / \mathrm{c}\right) \times \operatorname{Br}\left(\eta_{b} \rightarrow J / \psi J / \psi\right) \times\{B r(J / \psi \rightarrow \mu \mu)\}^{2}<2.6 \mathrm{pb}$, where $\eta$ is $\ln \left(\tan \left(\frac{\theta}{2}\right)\right)$ with $\theta$ the angle of the $\eta_{b}$ with respect to the beamline and $\mathrm{p}_{T}$ its transverse momentum.
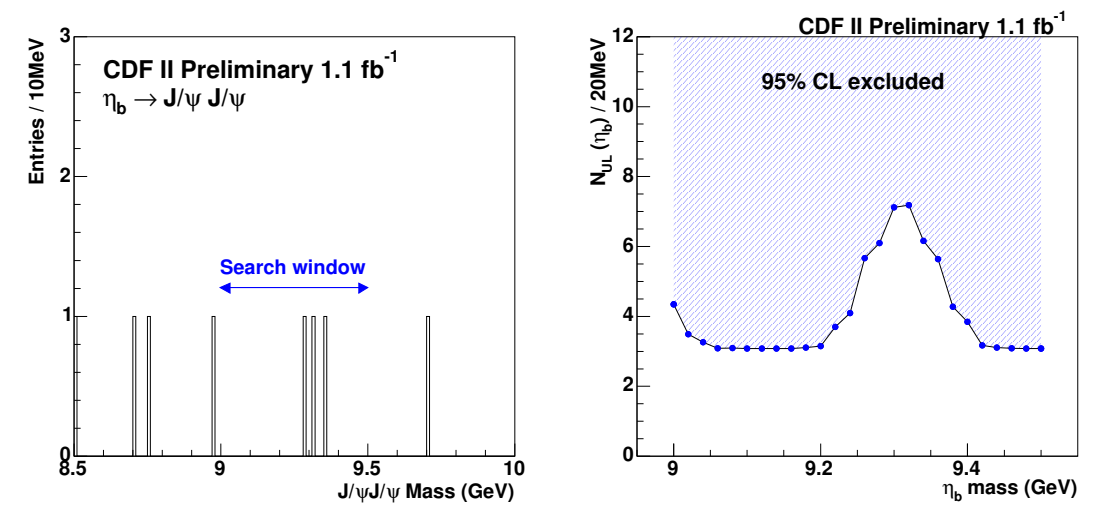

Figure 2: Mass Spectrum of $J / \psi J / \psi$ (left). Derived 95\% confidence limit on the number of $\eta_{b}$ (right).

\section{Study of orbitally excited $B_{s}$ and $B_{d}$ mesons}

Orbitally excited $B_{s}$ and $B_{d}$ mesons are studied at both Tevatron experiments [5][6]. There are four states for each meson; $B_{s(d) 0}^{*}$ and $B_{s(d) 1}$ with the orbital angular momentum $(\mathrm{L}=1)$ 


\begin{tabular}{|l|c|c|}
\hline Parameter $\left[\mathrm{MeV} / \mathrm{c}^{2}\right]$ & D0 & CDF \\
\hline $\mathrm{M}\left(B_{1}\right)$ & $5720.8 \pm 2.5 \pm 5.3$ & $5734 \pm 3 \pm 2$ \\
\hline $\mathrm{M}\left(B_{2}^{*}\right)$ & $5746.8 \pm 2.4 \pm 1.7$ & $5738 \pm 5 \pm 1$ \\
\hline $\mathrm{M}\left(B_{s 1}\right)$ & - & $5829.41 \pm 0.21 \pm 0.14 \pm 0.6$ \\
\hline $\mathrm{M}\left(B_{s 2}^{*}\right)$ & $5839.1 \pm 1.4 \pm 1.5$ & $5839.64 \pm 0.39 \pm 0.14 \pm 0.5$ \\
\hline
\end{tabular}

Table 1: Masses of orbital excited $B$ and $B_{s}$ mesons. The first given uncertainty is statistic, the second is systematic, and the third one, if given, is the mass uncertainty on the $B^{+(*)}$ mass.

and the spin of the light quark coupled to $\frac{1}{2}$, and $B_{s(d) 2}^{*}$ and $B_{s(d) 1}$ with an angular momentum of the light quark of $\frac{3}{2}$. The four states are commonly called $B_{s(d)}^{* *}$. In HQET the strong decay $B_{s(d)}^{* *} \rightarrow B^{+} K^{-}\left(\pi^{-}\right)$(The decay of $B_{s}^{* *} \rightarrow B_{s} \pi$ is forbidden by isospin conservation.) does not change the spin of the $b$ quark. Therefore both states with angular momentum $\frac{3}{2}$ for the light quark are expected to decay via $\mathrm{D}$-wave and to be narrow, while the other two decay via S-wave, which means they are broad. Broad states are very difficult to observe and therefore experimental studies are focused on the narrow states.

Conservation of angular momentum and parity allows for the $B_{s(d) 1}$ only the decay to the spin excited $B^{+*}$, while $B_{s(d) 2}^{*}$ can decay to both the ground state $B^{+}$and $B^{+*}$. The studies of $B_{d}^{* *}$ mesons are done at D0 (CDF) on $1 \mathrm{fb}^{-1}(370$ $\mathrm{pb}^{-1}$ ) of $p \bar{p}$ collision data, while both experiments use $1 \mathrm{fb}^{-1}$ for the $B_{s}^{* *}$ study. In Tab. 1 the results for measured masses in these studies are shown, in Fig. 3 the $B_{d}^{* *}$ spectrum of D0 and in Fig. 4 the $B_{s}^{* *}$ spectrum of CDF are shown. The $B_{d}^{* *}$ masses are not in good agreement, but contain still high uncertainties. Studies with more data are ongoing.

In the $B_{s}^{* *}$ sector both experiments agree on the $B_{s 2}^{*}$, for which LEP experiments had already evidence. CDF reports for the first time an observation of the $B_{s 1}$, which removes the ambiguity of assigning states to the resonances.

\section{$5 \quad$ First observation of charged $\Sigma_{b}^{(*)}$}

Another study [7] was recently finished at CDF on $\Sigma_{b}$ baryons. Up to now the only well established $b$-baryon was the $\Lambda_{b}$, which is an isospin singlet. The charged $\Sigma_{b}$ baryons containing bdd or buu decaying to $\Lambda_{b} \pi^{ \pm}$form the corresponding isospin triplet together with the neutral $\Sigma_{b}$,

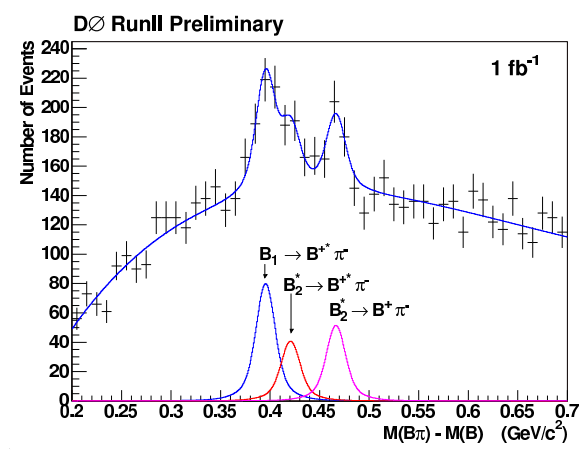

Figure 3: $B^{+} \pi^{-}$spectrum from D0.

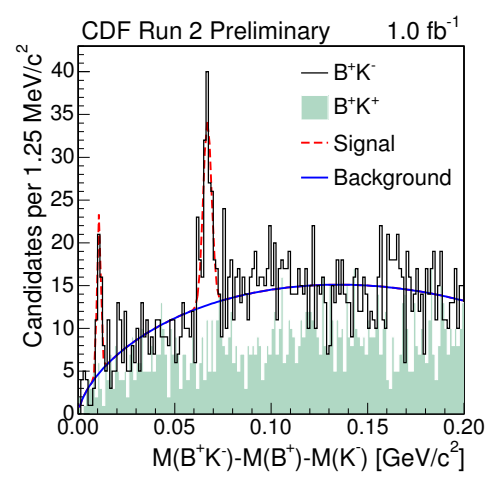

Figure 4: $B^{+} K^{-}$spectrum and $B^{+} K^{+}$ spectrum (filled area) from CDF. 
which is not studied, since neutral pions can't be detected at CDF. Based on a sample of about $3000 \Lambda_{b}$ 's in $\Lambda_{c}^{+} \pi^{-}$decay mode a blind search for charged $\Sigma_{b}^{(*)}$ is performed. Results for the masses of the $\Sigma_{b}$ ground states and of the spin exited states $\left(\Sigma_{b}^{*}\right)$ from a fit with widths fixed to predictions are shown in Tab 2. The obtained fit is shown in Fig. 5. The significance for all states together is higher than $5 \sigma$ and for each single state more than 3 $\sigma$ except of the $\Sigma^{+}$, which has $1.6 \sigma$ significance.

\begin{tabular}{|l|l|}
\hline $\mathrm{M}\left(\Sigma_{b}^{-}\right)$ & $5816_{-1.0}^{+1.0} \mathrm{MeV} / \mathrm{c}^{2}$ \\
\hline $\mathrm{M}\left(\Sigma_{b}^{+}\right)$ & $5808_{-2.3}^{+2.0} \mathrm{MeV} / \mathrm{c}^{2}$ \\
\hline $\mathrm{M}\left(\Sigma_{b}^{*+}\right)$ & $5837_{-1.9}^{+2.1} \mathrm{MeV} / \mathrm{c}^{2}$ \\
\hline $\mathrm{M}\left(\Sigma_{b}^{*+}\right)$ & $5829_{-1.8}^{+1.6} \mathrm{MeV} / \mathrm{c}^{2}$ \\
\hline
\end{tabular}

Table 2: The masses of the studied $\Sigma_{b}$ states with their statistical uncertainties. The systematic uncertainty is $1.7 \mathrm{MeV} / \mathrm{c}^{2}$ for each state.

\section{Summary}

As seen various $B$ states can be studied at the Tevatron on which other experiments can make only little or no contribution. We have seen the first precision measurement of the $B_{c}$ mass, which seriously probes the predictions from theory. Despite $\eta_{b}$ is not yet observed, studies cut already into its predicted parameter space. With the studies disentangling the narrow states of the $B_{d}^{* *}$ mesons and the first observation of $B_{s}^{* *}$ mesons, precise measurements are done, which can help to understand the QCD of a static color source. A contribution to this understanding is done as well by the new $\Sigma_{b}$ baryons, which reveal a bit

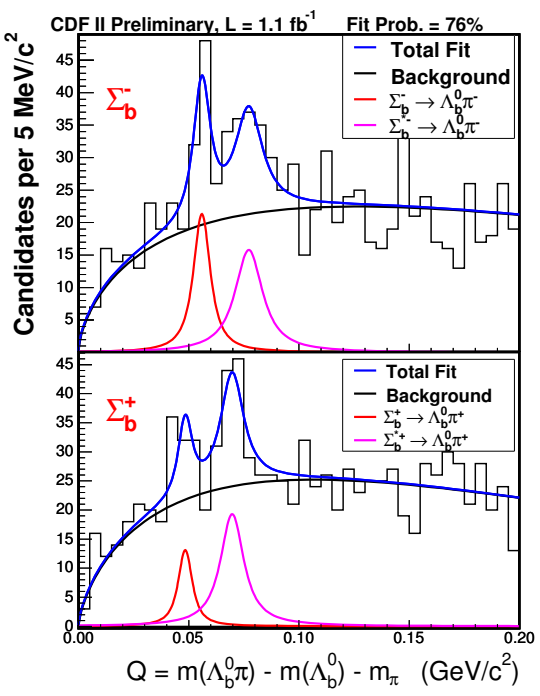

Figure 5: Mass Spectrum of $\Lambda_{b} \pi^{\mp}$. The lines show the full fit result, the background component and the signal component.

more of the largely unknown $b$-baryon sector. Since the Tevatron is running with higher luminosity than in years before, one can expect more contributions to the $b$-sector to come in the future.

\section{References}

[1] Slides: http: //indico. cern. ch/contributionDisplay . py? contribId=201\&sessionId=5\&conf Id=9499

[2] CDF Collaboration, F. Abe et al., Phys. Rev. Lett. 812432 (1998).

[3] CDF Collaboration, CDF note $\mathbf{8 0 0 4 .}$

[4] CDF Collaboration, CDF note $\mathbf{8 4 4 8 .}$

[5] DØ Collaboration, V. Abazov et al, arXiv:0705.3229v1 [hep-ex]; CDF Collaboration, CDF note 7938.

[6] CDF collaboration, CDF note 8468; D $\varnothing$ collaboration, D $\varnothing$ note $\mathbf{5 0 2 7 .}$

[7] CDF Collaboration, CDF note $\mathbf{8 5 2 3 .}$ 